\title{
Traduire
}

Revue française de la traduction

$220 \mid 2009$

Organisations internationales | Bicentenaire de Louis

Braille

\section{La traduction au sein d'une organisation internationale : témoignages}

\section{Christine Cross}

\section{(2) OpenEdition \\ Journals}

Édition électronique

URL : http://journals.openedition.org/traduire/380

DOI : $10.4000 /$ traduire.380

ISSN : 2272-9992

Éditeur

Société française des traducteurs

Édition imprimée

Date de publication : 15 juin 2009

Pagination : 36-38

ISSN : 0395-773X

Référence électronique

Christine Cross, «La traduction au sein d'une organisation internationale : témoignages », Traduire [En ligne], 220 | 2009, mis en ligne le 12 novembre 2013, consulté le 01 mai 2019. URL : http:// journals.openedition.org/traduire/380 ; DOI : 10.4000/traduire.380 


\section{La traduction au sein d'une organisation internationale : témoignages}

\section{Christine Cross}

La traduction fait partie de ces rares activités professionnelles que l'on peut exercer sans aucune qualification formelle. Le métier de traducteur peut se pratiquer aussi bien en entreprise qu'en profession libérale mais, à la différence du secteur médical, par exemple, où quel que soit son statut (libéral ou hospitalier), le médecin doit être titulaire de certains diplômes, le traducteur peut ne jamais avoir suivi d'études de traduction, à partir du moment où il maîtrise (ou pense maîtriser !) suffisamment bien une $2^{\mathrm{e}}$ langue.

Force est de constater cependant que, lors de l'embauche, les organisations internationales manifestent depuis quelques années une nette préférence pour les diplômés des écoles de traduction, surtout ceux qui ont déjà effectué des stages dans leurs murs.

II existe d'ailleurs d'importantes différences entre travailler au sein d'un service de traduction et exercer son métier en tant qu'indépendant. Dans le dernier cas, le traducteur est, en règle générale, seul face à son ordinateur, ne connaît pas toujours son client final mais, par contre, dispose librement de son temps. En revanche, le traducteur salarié fait normalement partie d'une équipe et doit respecter certaines contraintes imposées par son employeur (lieu et horaires de travail...).

Prenons l'exemple d'une ex-collègue qui travaille désormais pour le compte d'un de ces tribunaux internationaux siégeant aux Pays-Bas. Ce genre de poste est très convoité, et ceci pour de nombreuses raisons. Comme elle le dit elle-même : "Tout d'abord il y a les aspects financiers : salaire, statut fiscal, caisses de prévoyance et autres avantages en nature. Ensuite, les grandes organisations comme les tribunaux disposent de moyens importants, ce qui leur permet d'équiper leurs traducteurs de matériel informatique performant et de logiciels dédiés. Vient se greffer là-dessus la possibilité d'accéder à des banques de données techniques et d'avoir des contacts directs avec des experts dans les disciplines concernées au sein de l'organisation. Autre élément important, les formations dont ils peuvent bénéficier avec, à la clé, la perspective d'un avancement hiérarchique ". Le seul bémol à ses yeux : l'impossibilité d'utiliser toutes ses combinaisons linguistiques et le fait d'être cantonnée à un rôle de traductrice, elle qui autrefois alliait traduction et interprétation. 


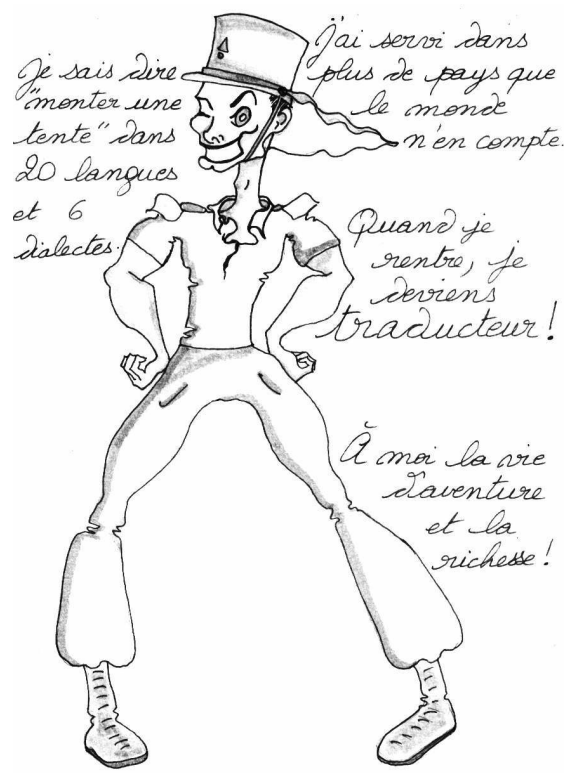

Illustration : Marlène Junius, http://alotoftralala.over-blog.com

Questionnée sur l'organisation du service de traduction, elle explique : "Chez nous, on distingue 2 catégories: traducteur et réviseur, ces derniers étant des traducteurs plus gradés du fait de leur ancienneté dans la maison. Un réviseur passe le plus gros de son temps à contrôler le travail de ses collègues traducteurs, y compris celui des traducteurs indépendants auxquels nous faisons parfois appel. Soit dit en passant, beaucoup de nos documents étant confidentiels, nous ne pouvons les confier à l'extérieur. Au sein de l'équipe, les traducteurs les plus expérimentés, quant à eux, ne sont pas nécessairement obligés de faire relire leurs textes, sauf dans le cas de documents " sensibles". Mais, si un texte ne passe pas entre les mains d'un réviseur, il devra au minimum être relu par un pair, ne serait-ce que pour en éliminer les éventuelles coquilles...".

Le réviseur note les modifications qu'il estime nécessaires et retourne le texte à son traducteur, qui doit ensuite passer en revue les changements proposés tout en les intégrant dans le texte. L'objectif de cette procédure est de lui donner l'occasion de réfléchir aux corrections apportées par son réviseur et de les retenir plus facilement grâce à l'effort de transcription qu'il doit fournir. Et notre interlocutrice ajoute : "L'enchaînement le plus long dans ces divers processus d'échange se trouve dans le cas d'un travail qui passe du traducteur au réviseur, revient vers le premier en vue de son adaptation, subit ensuite une relecture de contrôle avant de revenir enfin chez le traducteur, ce dernier étant toujours responsable de l'intégration des modifications proposées".

A l'heure actuelle, le service de traduction ne dispose ni de logiciel de traduction automatique ni de base de données terminologique centrale. Il est prévu à court terme cependant de l'équiper d'un outil d'aide à la traduction, l'objectif essentiel étant de maintenir une bonne cohérence terminologique et de garantir l'exactitude des citations relevées dans d'autres textes officiels. Pour notre interlocutrice, un tel système pourrait éventuellement également permettre des gains de productivité mais " ce n'est pas le but de l'opération ".

Ensuite elle nous décrit une journée typique dans la vie d'un traducteur/réviseur : 
"Tous les matins, grâce à un système de gestion informatisé, les traducteurs sont informés par messagerie des tâches qu'ils devront accomplir ainsi que de la nature de ces tâches (traduction, révision, relecture...). On leur indique le nom du document à traiter, le nombre de mots ainsi que le délai qui leur est accordé. Dans un premier temps, ils devront rechercher la traduction exacte des citations, titres et autres abréviations figurant dans leur texte. Pour cela, ils pourront consulter des sites web, les différentes bases de données réparties à travers l'organisation, les dictionnaires dont ils disposent, le service documentation de la maison, voire même l'auteur du texte. S'ils se retrouvent face à des difficultés d'ordre logistique, ils devront contacter la personne chargée de la coordination, qui pourra alors soit répartir le travail entre plusieurs traducteurs (internes ou externes) pour garantir le respect du délai fixé, soit négocier une prolongation de délai. Sinon, le cycle suit son cours habituel... En tout état de cause, tous les traducteurs doivent systématiquement respecter l'accord de confidentialité signé au moment de leur embauche ".

Ce modèle est en fait très courant dans les organisations internationales, comme peut l'attester l'auteur de cet article, qui a travaillé pendant plusieurs années au sein d'une association professionnelle internationale!

Quelles conclusions tirer de cette mini-présentation ? Premier constat : un jeune diplômé qui débute a tout à gagner en choisissant de faire son apprentissage en "entreprise " (dans le sens le plus large du terme). II sait qu'il sera entouré de personnes expérimentées, qui pour-

Votre mission si vous l'acceptez :

- 500000 mots tamoul-wolof pour dans 1 heure

- arroser les plantes

- dire bonjour à vos collègues

Attention, cet ordinateur s'autodétruira dans 5 minutes.

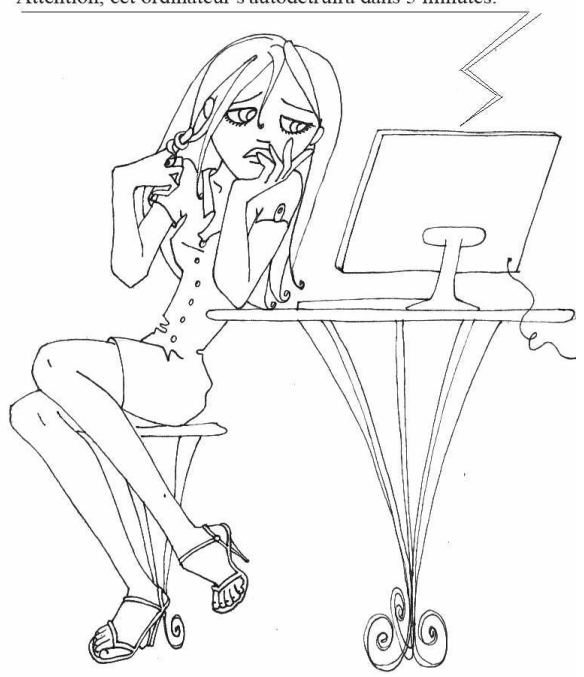

Illustration : Marlène Junius, http://alotoftralala.over-blog.com ront l'aider à trouver ses marques et gagner ses galons de noblesse. De surcroît, il pourra être en prise directe avec ses clients dans le cas où il aurait besoin d'éclaircissements ou d'explications de texte. II sait également que son travail sera relu par des collègues plus anciens, ce qui ne manquera pas de lui être utile. En revanche, il sait aussi qu'il devra respecter la hiérarchie et accepter de travailler en équipe, avec tout ce que cela implique.

Et c'est précisément cette absence d'autonomie qui, pour certaines personnes, peut s'avérer rédhibitoire. Reste alors la voie de la traduction indépendante... 